\title{
Edificio SOMISA: Entre los elementos y la totalidad
}

\author{
Building SOMISA: Between the elements \\ and the totality
}

Ju

$<$ Resumen>

El edificio SOMISA ubicado en Buenos Aires, proyectado por Mario Roberto Álvarez a fines de los 60 y terminado de construir en 1977 . es en el contexto argentino un ejemplo único de arquitectura de acero y vidrio. Existen varios motivos para considerar sus aportes patrimoniales, teórico-arquitectónicos y técnicos.

$<$ Abstract>

The SOMISA building, located in Buenos Aires and designed by Mario Roberto Alvarez at the end of sixties is, in an Argentine context, a unique example of steel and glass architecture. There are various reasons to consider its patrimonial values, the theoretical consequences and the technical effects of the building.

<PALABRAS CLAVE >

PROYECTO / COMPOSICIÓN / TOTALIDAD / CIUDAD

-KEY WORDS>

PROJECT / COMPOSITION / TOTALITY / CITY
Aspectos generales de la obra de Mario Roberto Ál varez ${ }^{1}$

La mayor parte de los edificios de Álvarez responden a composiciones unitarias en las que las partes espaciales se presentan reconocibles, con un principio y un fin. Sus edificaciones correspondientes a vivienda y oficinas en altura, responden a tipos de lote de cuadra o de esquina impactados por la estructura formal de torre o de torre con basamento, también a lotes más amplios en los que combina la torre exenta y un tipo de viviendas en altura recostado sobre una medianera o solamente la torre exenta. Cuando desarrolla edificios en altura de carácter más monumental emplea estructuras más expresivas y referibles a conjuntos de volúmenes puros adaptados a las posibilidades del lote, lo que implica el empleo de tipologías urbanas que gradúan la referencia exenta de la estructura formal desde situaciones estrechas de cuerpos separados por patios, situaciones intermedias de volúmenes recostados sobre la

\footnotetext{
Arquitecto. Profesor Titular Historia Universidad de Concepción del Uruguay, Entre Ríos, Argentina.

Arquitecto argentino (1913 Buenos Aires). Termina sus estudios de arquitectura en 1936, estableciendo en el año 1947 la oficina Mario Roberto Álvarez y Asociados. Ha recibido numerosos premios, sus obras han sido exhibidas en diversas exposiciones en Argentina y en el exterior. Miembro de la Academia Nacional de Bellas Artes, Argentina.
} 
medianera con volúmenes menores adosados hasta situaciones totalmente exentas de dos o más volúmenes articulados.

En general los edificios de Álvarez desarrollan sus elementos de arquitectura como una «consecuencia natural» del planteo compositivo. La geometría pura y regulada que organiza el o los volúmenes se descompone en sus elementos primarios, planos y líneas, los cuales permanecen sujetos al volumen virtual de referencia sin dar lugar a la aparición de elementos ajenos a esta realidad geométrico-formal. Solamente en los casos en que la presión funcional y monumental del tema es muy importante, Álvarez está dispuesto a incorporar detrás de una segunda línea espacial «volúmenes puros» y sin descomponer que expresan la carga funcional y monumental del tema. La expresividad de la particularidad en Álvarez es algo sistemáticamente anulado. El caso del edificio SOMISA se aleja de esta tendencia de claridad compositiva y serenidad expresiva de sus elementos de arquitectura. No surge en forma inmediata una respuesta a esta anomalía en una producción arquitectónica de más de 60 años, aunque se tratará de encontrar alguna explicación en el curso del presente trabajo, el que parte por reconocer que sin lugar a dudas este edificio de valor monumental y patrimonia para la Argentina se constituye en uno de los ejemplos más curiosos e interesantes de la obra construida de Mario Roberto Álvarez.

\section{Ргimera cuestión}

Este edificio suscita una necesaria reflexión respecto de la relación entre las partes que lo componen y el resultado final o totalidad resultante. El problema de las partes y su correspondencia con un todo es en gran medida un argumento crítico indispensable en todas las arquitecturas que se pretendan racionalistas. Respecto de esto aclara Marcelo A. Trabucco en su libro « ¿De la Totalidad a las Partes?»:

«Desde el punto de vista de la crítica arquitectónica y, sobre todo en los enfoques tradicionales de la historia de la arquitectura, se hace evidente que el concepto de totalidad arquitectónica adquiere jerarquía dominante sobre los posibles conceptos de partes que intervienen en su configuración... La lectura crítica o interpretativa del todo se encara pensando en que éste es el que le confiere sentido al discurso arquitectónico y,

Trabucco, M.A. ¿De la totalidad a las partes? Buenos Aires: Editorial de Belgrano, 1999.
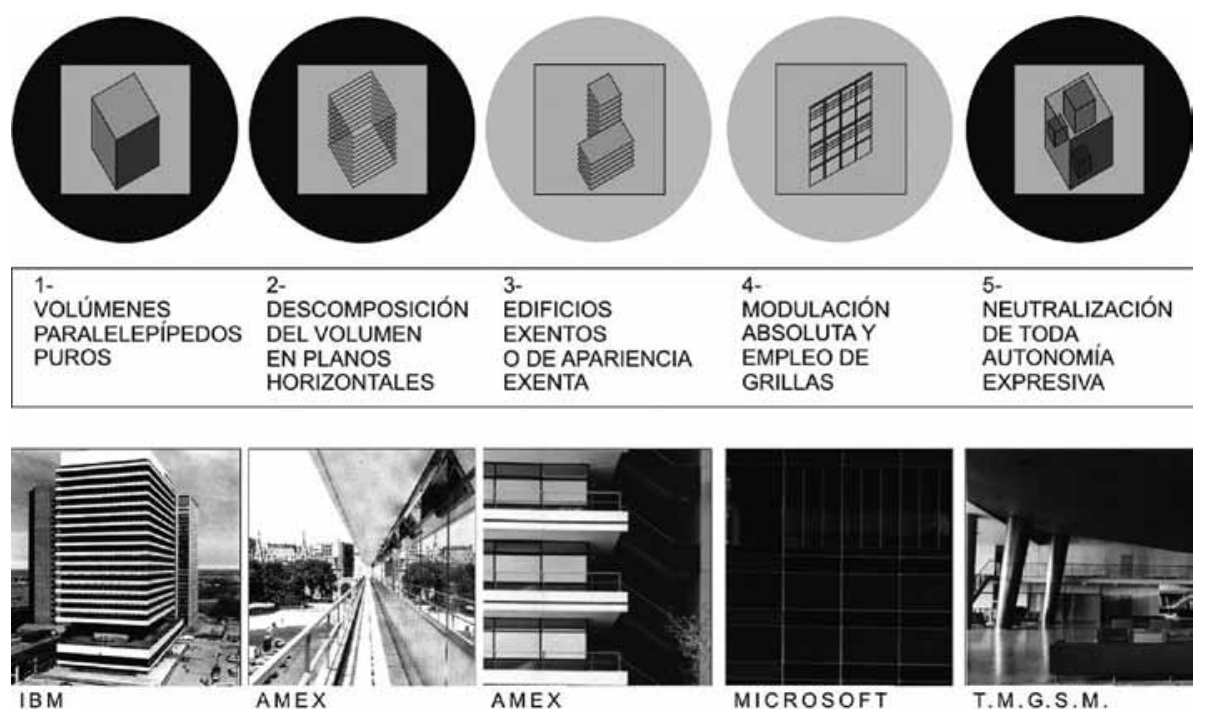

Figura 1.

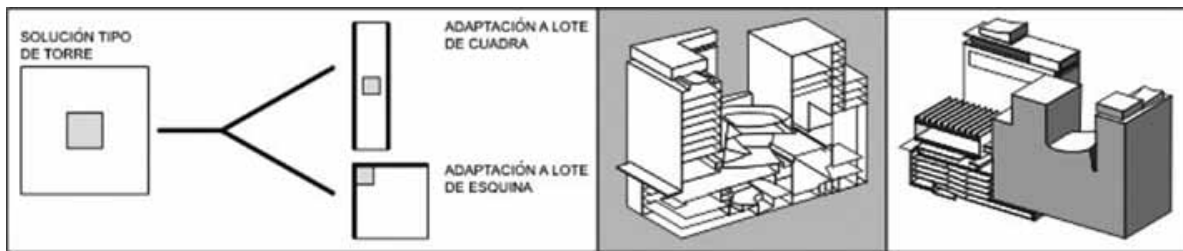

Figura 2

Tipos de lote impactados por la estructura formal de torre, lotes estrechos de cuerpos y patios y lotes mayores con un volumen sobre la medianera y uno menos adosado.
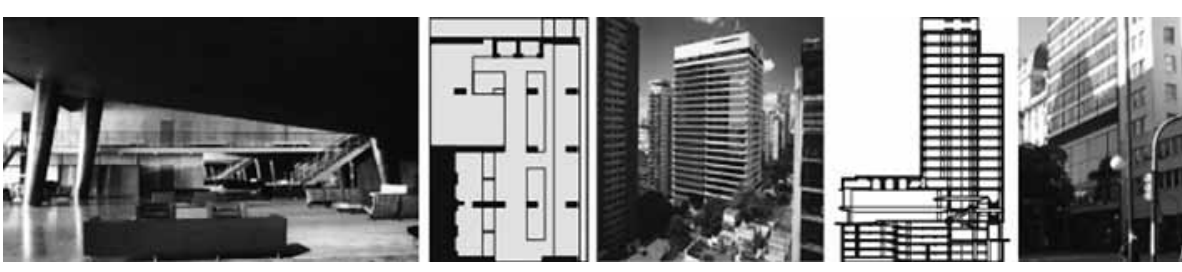

Figura 3 .

Foyer del T.M.G.S.M. Planta UB c/ascensores en negro y fachada posterior. Corte Bolsa de Comercio y fachada L.N. Alem.

consecuentemente, que el papel que le cabe a las partes es el de celebrar dicho sentido por medio de afirmaciones parciales que coinciden, o por lo menos son coherentes, con dicho sentido general».

EI SOMISA es un edificio en el que se vuelve difícil terminar de definir una realidad compositiva o estructura formal clara. Si se observa desde la Diagonal Sur se percibe una masa continua que celebra la cuadra, si se observa desde la esquina, se percibe un elemento vertical que parece exento, y esto se nota aún más si se observa desde la avenida Belgrano. Si se pretende separar dos elementos, uno de continuidad con la diagonal 
y otro exento tipo torre, resulta imposible. El SOMISA no es el resultado de la articulación compuesta de dos elementos con funciones diferentes; pero sin embargo la percepción de mismo alude ambas articulaciones y ambos elementos. Esta ambigüedad es la que desde el punto de vista de los enfoques tradicionales de la crítica arquitectónica, constituye un híbrido que destruye la secuencia jerárquica entre el todo y las partes.
La aparición y el desarrollo de la arquitectura moderna y afines en Buenos Aires, como en otras ciudades, debió adaptarse a una realidad compuesta por capas o estratos de realidades acumuladas que construyeron un fenómeno de densificación cultural que exige que cualquier manifestación que en él desee desarrollarse deba considerarlo ${ }^{3}$. El caso que nos ocupa define un sector o punto neurálgico cargadísimo
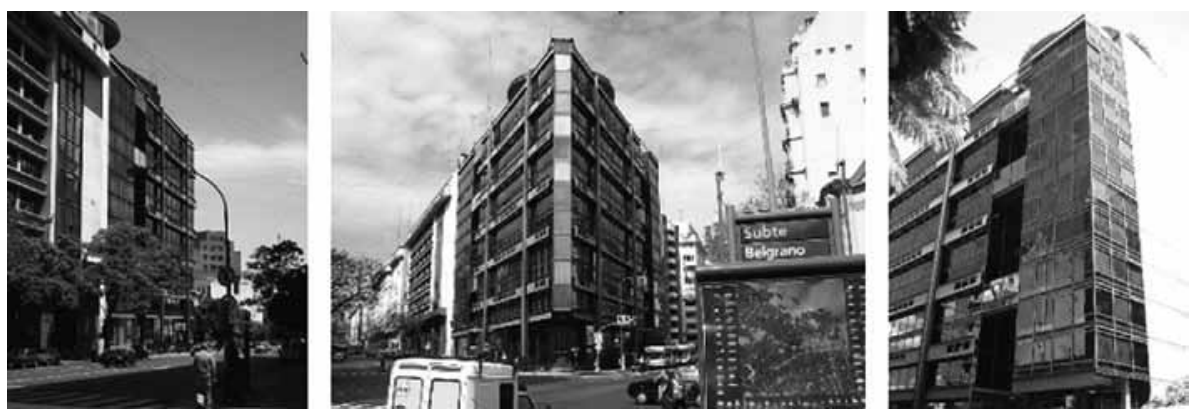

Figura 4

Variabilidad compositiva: ocupación de perímetro, †orre de esquina y edificio exento.
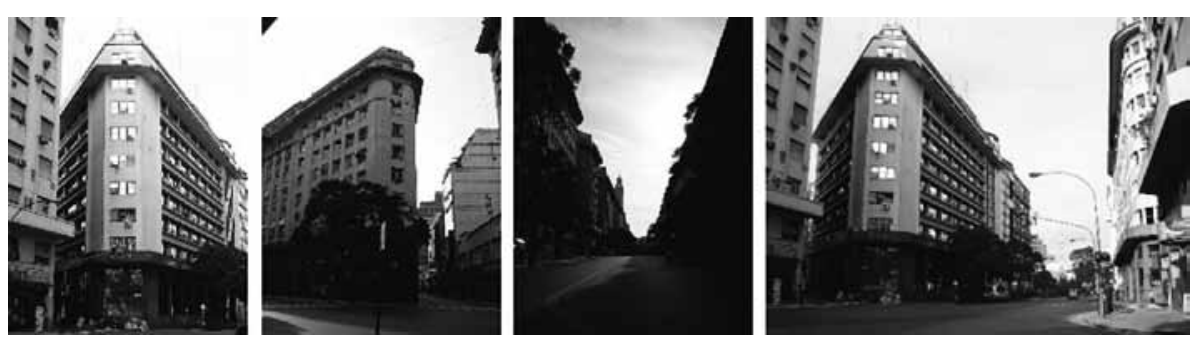

Figura 5

Diagonal Sur: dos esquinas, foto en dirección a plaza de mayo y foto en dirección a Avenida Belgrano.
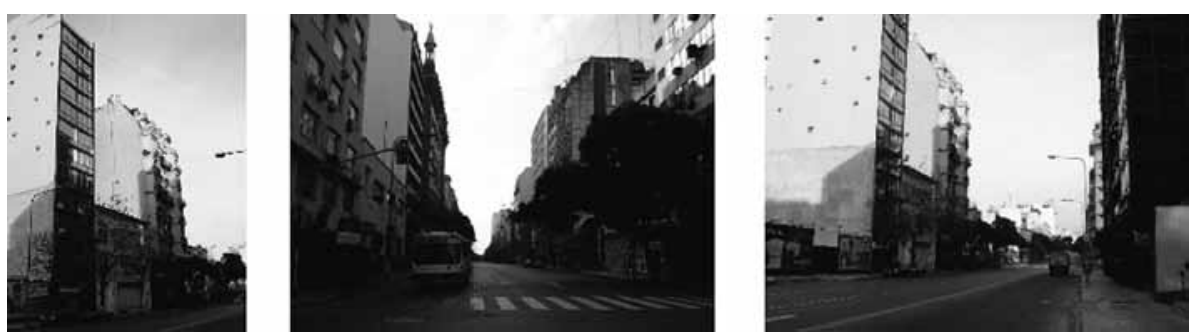

Figura 6

Avenida Belgrano: tejido discontinuo, foto en dirección a L.N. Alem y foto en dirección a la 9 de Julio.

de esta densificación. El encuentro entre ambas avenidas, sella el contacto entre dos formas distintas de crecimiento y desarrollo urbano. La diagonal sur representa una visión haussmanniana ${ }^{4}$ y la avenida Belgrano y el tejido circundante forman parte de esa realidad acumulativa y tradicional de tipos que fueron completando la ciudad. En este caso, la Diagonal Sur termina en Avenida Belgrano, ya que se tornó imposible seguir adelante con el objetivo inicial, que era hacerla llegar hasta la 9 de Julio. La iniciativa que impulsa el concurso del edificio SOMISA forma parte de un tercer estrato en el que los objetivos tardíos de modernidad y monumentalidad unidos al poder estatal dan lugar a la posibilidad de llevar adelante el concurso y la construcción de un monumento administrativo de la industria nacional; en este caso el de una empresa dedicada a la producción de acero. Existe finalmente un cuarto estrato que es el de los valores y paradigmas modernos referenciales del arquitecto. Estas cuatro capas o estratos están presentes en el edificio formando parte de las exigencias implícitas que aunque prácticamente imposibles de conciliar en una síntesis racional, igualmente fueron incorporadas aceptándose que el edificio se aleje de esa síntesis racional, motivo por el cual la interpretación del mismo se funde en muchas direcciones.

\section{La Diagonal}

La Diagonal Roque Sáenz Peña o Diagonal Sur es parte de una intervención urbana desarrollada a inicios del siglo xx con el objetivo de integrar el sector céntrico de la ciudad. Esta diagonal orientada hacia el sudoeste creció menos que la Diagonal Norte, su correspondiente simétrica respecto del eje Casa Rosada-Congreso definido por la Avenida de Mayo. El sistema de diagonales prevé un código formal que controla las alturas, remates, ritmos horizontales, etc. Parte importante de este código está presente en las bases del concurso, obligando a generar una propuesta acorde con la continuidad de la diagonal.

\section{Avenida Belgrano y trama urbana}

Forma parte de la trama de ensanches de avenidas correspondientes a una trama urbana que las ubica cada cuatro cuadras. El damero de origen colonial llega a este sector sin alteraciones en su ortogonalidad y la zona

Diez, F. Buenos Aires y algunas constantes en las transformaciones urbanas. Buenos Aires: Editoria UB, 1996

Referido a Georges Eugène Haussmann 
ha pasado por sucesivas consolidaciones de densificación creciente. La relación con la avenida es más flexible, optando Álvarez por definir una separación del edificio respecto de la medianera del lote colindante.

\section{El lote}

La asimetría que se verifica entre las dos avenidas, también está presente en el lote, cuya apariencia de triángulo regular truncado en la esquina, se distorsiona a medida que se extiende, dando lugar a un área trapezoidal irregular. Esta asimetría del lote también implicará una repartición de profundidades diferentes que influirán mucho de la zonificación de vacíos, accesos y áreas principales y secundarias.

\section{Las exigencias del concurso}

Una de las exigencias de concurso más importantes y decisivas es la obligatoriedad del empleo de acero, material producido por la empresa. Es importante tener presente que los sistemas de perfilaría laminada son estrictamente un alarde técnico de lo que la industria podía realizar en ese momento. La otra exigencia importante y ya mencionada es la del requisito de respetar el conjunto de normas que regulan el límite de fachada contenidas en el código de la Diagonal Sur.

\section{Valores y paradigmas del arquitecto}

Los ideales modernos que se comprueban en la mayor parte de la obra de Álvarez responden a una concepción arquitectónica que toma partido por la forma pura, reconocible y cristalina. En estas estructuras formales los elementos se mantienen jerárquicamente unidos a los volúmenes principales manteniendo una secuencia jerárquica de total subordinación de las partes al todo. Como esta manera de concebir la arquitectura no esta presente en el SOMISA surge la necesidad inmediata de explicar cuales son las causas que provocaron este fenómeno y cómo entra este caso dentro de una concepción más amplia de los valores y paradigmas arquitectónicos de Álvarez.

El concepto espacial de la arquitectura que ocupa la línea municipal y que constituye

Georges Eugène Haussmann. Político y urbanista francés (1809-1891). Prefecto de París, entre los años 1853 y 1870. Desde este cargo, emprendió una profunda remodelación de París, otorgándole a la ciudad su fisonomía actual. una realidad de perímetro es diferente del concepto espacial de la arquitectura exenta tipo torre, ambas estructuras espaciales resultan incompatibles desde el punto de vista de la concreción de un proyecto racional. En este caso entran en conflicto los requisitos del concurso respecto del respeto de la continuidad urbana de la diagonal con las expectativas de modernidad implícitas en el tema y los valores y paradigmas del arquitecto. El lote posee una geometría y extensión que complican una ocupación regular. Si se analiza la geometría trapezoidal a que pertenece y se considera la experiencia del sistema de loteos desarrollado por Haussmann ${ }^{5}$ en París, se comprueba que el lote debería ser simétrico respecto de la generatriz del bloque.
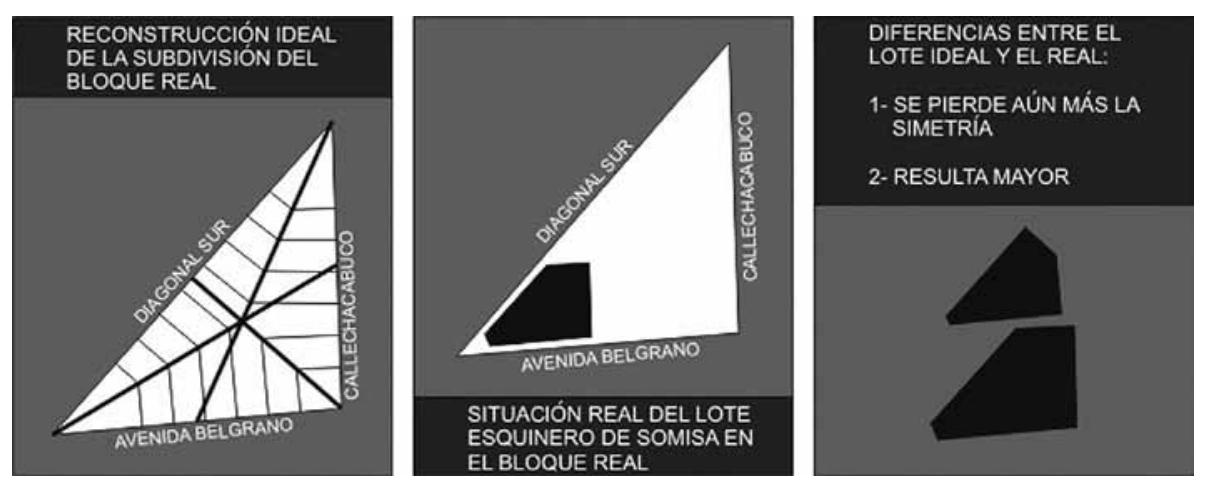

Figura 7

Reconstrucción ideal de la subdivición del bloque real Situación real del lote esquinero de Somisa en bloque real. Diferencias entre el lote ideal y el real 1) Se pierde aún más la simetría; 2) Resulta mayor.
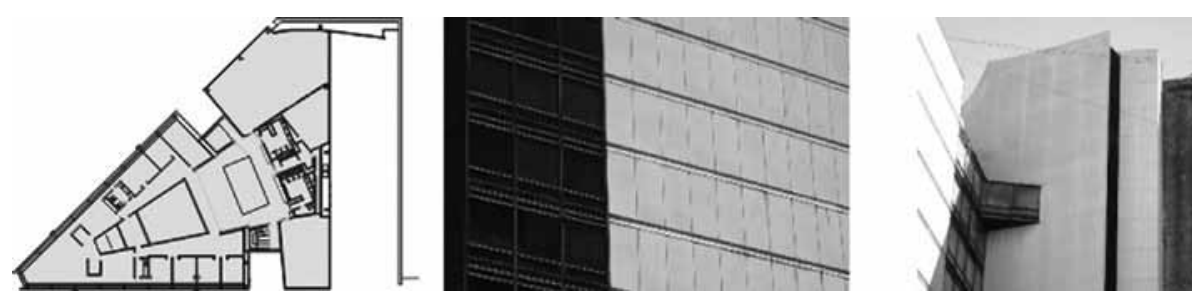

Figura 8

SOMISA: Planta tipo, muro en Chanfle y Poché
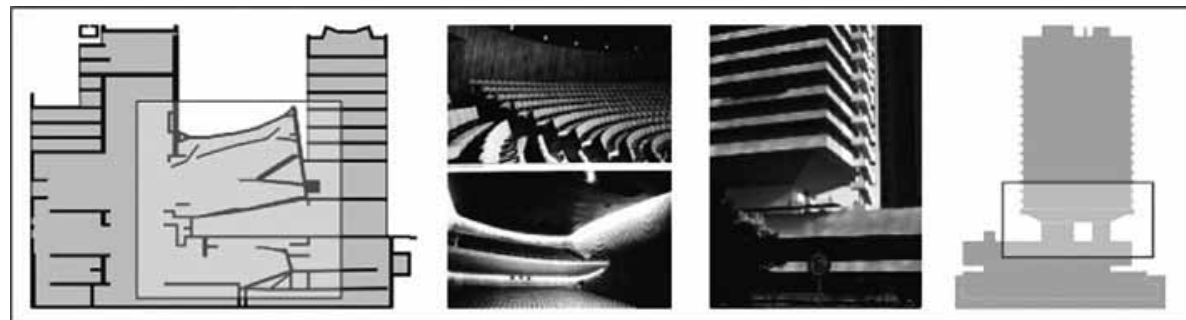

Figura 9 .

Corte Teatro Municipal General San Martín, salas del teatro IBM y corte del IBM. 
Álvarez decidió dejar en chanfle los espacios de la estructura formal del edificio a medida que se acercan a este límite de las medianeras con sus lotes vecinos. Ese límite o chanfle fue materializado por una pared paralela a la línea divisoria con el lote lindero sobre Avenida Belgrano y el borde ahora al descubierto de la línea divisoria con el lote lindero de Diagonal Sur fue «recubierto» con un tratamiento "poché» que esconde un pleno y en definitiva la inexistencia de espacio habitable detrás de él. Se comprueba la adversidad del lote para absorber las diferencias entre las dos avenidas.

\section{Segunda cuestión}

Continuando con la disquisición acerca de la relación compleja entre las partes y el todo, en esta segunda parte se tratará de explicar el por qué del sistema de acero, cuya realidad tecnológica lo tiende a volver un sistema de elementos, adecuado para una arquitectura elementarista. Álvarez suele construir sus edificios con estructura de hormigón armado. El empleo de este sistema constructivo en Argentina es el más habitual para edificios de altura, por lo tanto la racionalidad del material y la lógica o sentido común de su empleo en la Argentina son argumentos suficientes para entender por que fue adoptado por Álvarez. Sus edificios hechos en hormigón armado fueron trabajados de manera tal que las columnas fueran las mínimas necesarias y las vigas y losas se puedan asociar al sistema de planos formal que compone el edificio.

De esta manera la estructura prácticamente perdía poder expresivo y se fundía con la forma geométrica de la estructura formal. Para Álvarez la estructura no suele ser un tema expresivo de por sí.
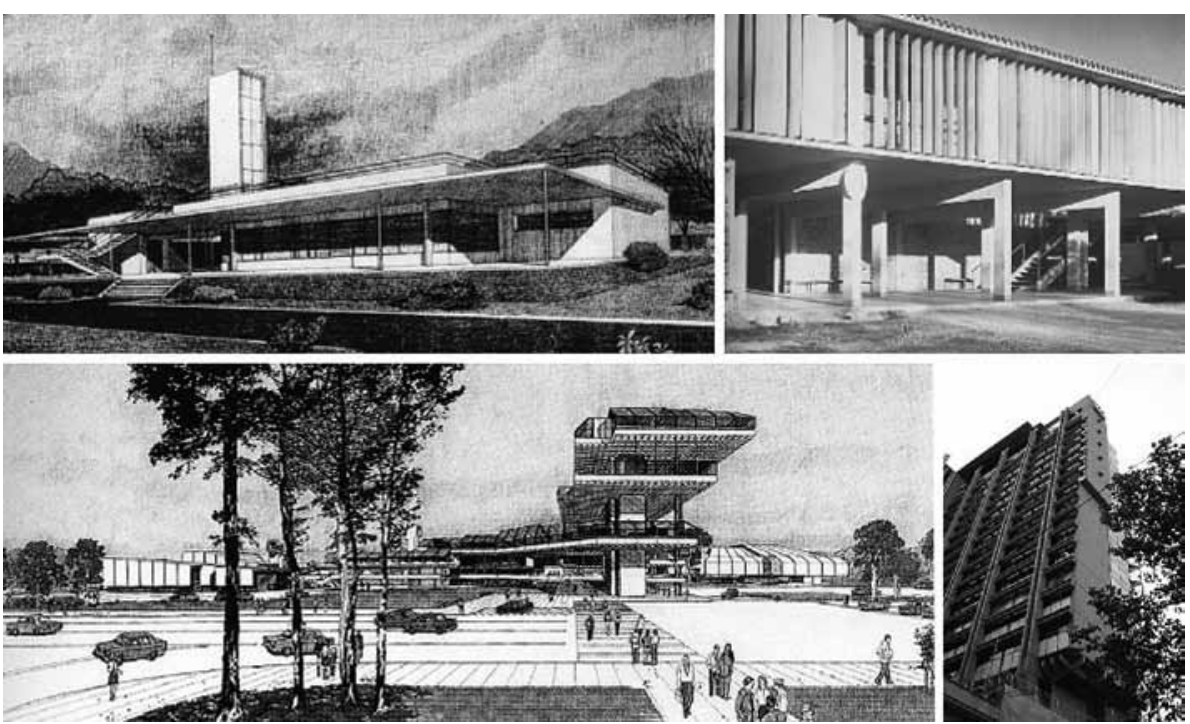

Figura 10

Restaurante Rocantti (Pergamino). Centro asistencia de Jujuy. Proyecto Universidad Nacional de La Plata. Sanatorio Guemes.
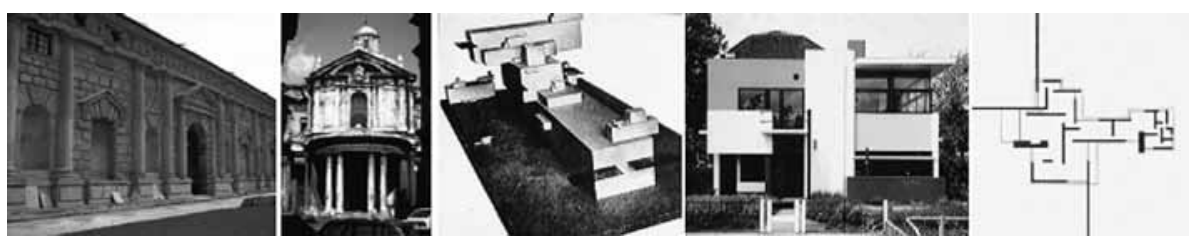

Figura 11

Palacio de Té. G. Romano, Sta. María de La Pace. D. Da Cortona, mauqeta. Malevich, Casa Schroeder. G. Rietveld, casa de ladrillos. Mies van der Rohe.
En este contexto de neutralidad estructural, el SOMISA produce serias dudas, con sus elementos estructurales y de cerramiento que adquieren una intensidad expresiva sobresaliente. Pareciera que en este caso, los ideales modernos de Álvarez hubiesen experimentado un alejamiento de la concepción más purista y volumétrica para pasar a experimentar caminos coincidentes con las arquitecturas de la vertiente elementarista y expresiva. Si se consideran algunas de sus primeras obras, particularmente el restaurante Roncatti en Pergamino, los proyectos de universidades y algunos casos puntuales como el Hospital Güemes, se puede reconstruir una línea de exploración elementarista cargada de una expresividad de rasgos tecnológicos y formales que habitualmente no suelen considerarse en su obra. Respecto de esta línea de expresión próxima a las experiencias constructivistas o neoplásticas, Francisco Bullrich ${ }^{6}$ dijo:

"...en estos proyectos, un riguroso planteo modular, precedido de un estudio sistemático, se asocia a una libre organización del espacio y a una estructuración volumétrica más suelta que por momentos parece recoger el eco de la imaginativa tradición constructivista».

El empleo de un sistema elementarista hace que la expresión repose sobre los elementos antes que sobre la obra total. Existe una tradición arquitectónica que propuso a lo largo de la historia la posibilidad de sustentar el orden arquitectónico sobre la coherencia del sistema de elementos de arquitectura. En esa tradición elementarista se inscriben arquitecturas tan variadas como las que produjeron arquitectos manieristas como Giulio Romano, arquitectos barrocos como Domenico da Cortona en Santa María de La Pace y arquitectos modernos como los constructivistas rusos, los elementaristas de De Stijl, las obras previas a la segunda guerra de Mies Van Der Röhe, la obra de postguerra de Richard Neutra, etcétera.

La estrategia elementarista posee la capacidad de reencarrilar la comprensión de edificio aceptando la condición híbrida de su composición. De esta manera las lecturas parciales no se anulen en una referencia general o totalidad que imponga un juicio inmediato que ponga en evidencia las incompatibilidades existentes. Probablemente y conjuntamente con los requisitos del concurso, esta realidad elementarista y expresiva tenga esa función, desviar el

Bullrich, F. «El poder de la síntesis». Revista Summa $1987 ; 233$ y 234 
discurso de la racionalidad compositiva adoptando un nuevo discurso situado en el campo de la racionalidad elementarista. Pero también, además de ser oportuna esta estrategia elemantarista, es posible asociarla con otros edificios construidos o proyectados por Álvarez, que aunque no se realizaron en acero, igualmente poseen ese empleo jerárquico de los elementos por sobre la jerarquía de la totalidad. Existen algunas opiniones que parecen coincidir con esta hipótesis, como la opinión de Bullrich anteriormente citada, también existen algunos proyectos y edificios construidos por Álvarez que siguen esta línea (el restaurante Roncatti, las universidades de fin de la década del 60, el hospital Güemes y el SOMISA). Finalmente el mismo Álvarez reconoce que su formación fue inclusiva y no exclusiva, sugiriendo la posibilidad de encontrar en su propia obra más influencias que las habitualmente reconocidas ${ }^{7}$ :

"En fin, de influencias, las tengo todas. Esto no es original mío, creo que lo dijo Borges: “iinfluencias?, todas». Mentiría si dijera que no admiro a Neutra; o al vienés Harry Siedler».

Considerando lo dicho por Bullrich, arquitecto que conoció y trabajó con Álvarez, considerando lo dicho por el mismo Álvarez y tomando en cuenta el rastreo de ciertas obras «menos representativas de la arquitectura típica de Álvarez» es posible detectar una línea de acción proyectual que aunque fragmentaria actúa en forma pendular respecto de su obra más representativa. En general estas obras o a veces episodios parciales de edificios (sucesión de salas en el Teatro Municipal General San Martín) suelen apoyarse en el poder de los elementos de naturaleza tecnológica; en Álvarez es nula la posibilidad de encontrar episodios elementaristas puramente formales.

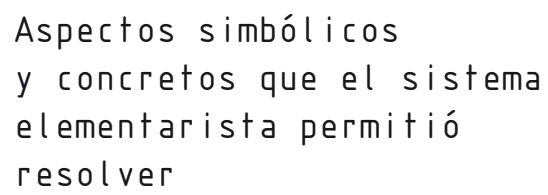

Continuidad y desarrollo de la morfología urbana proveniente de la Diagonal Sur

Piñón, H. Mario Roberto Álvarez y Asociados. Barcelona: Edición UPC, 2002.
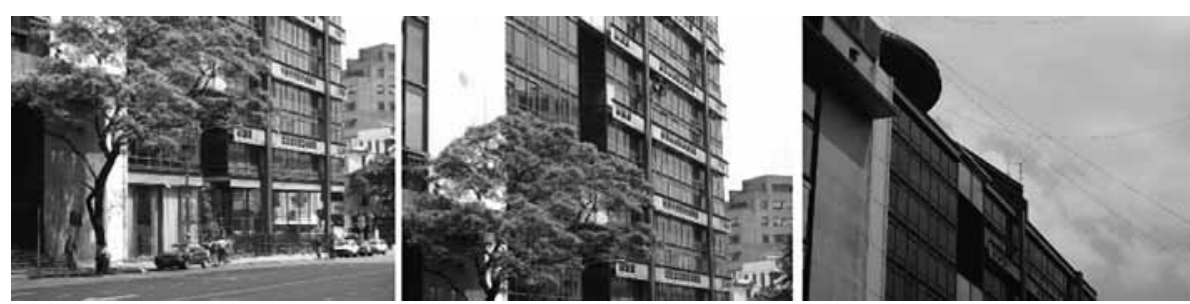

Figura 12A. Edificio SOMISA: Nivel de acceso, plantas intermedias y volúmenes de remate en la †еггaza.
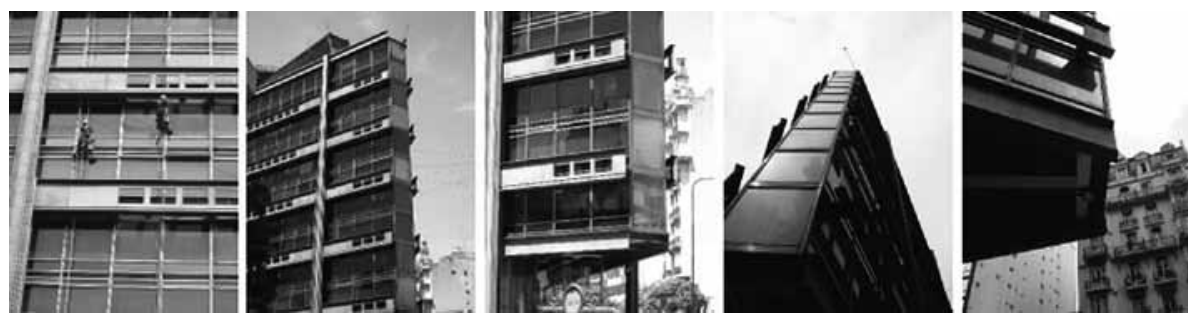

Figura 12B

Edificio SOMISA:Estructura y cerramiento y fotografías de la esquina
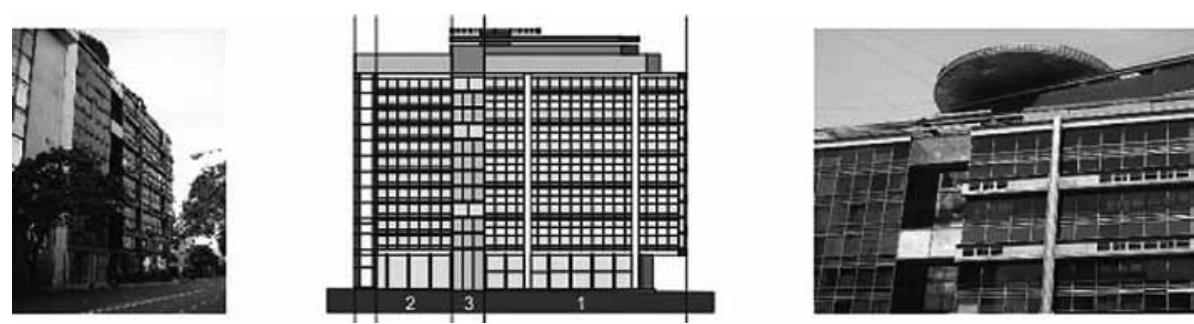

Figura 13

Fotografía del frente sobre Diagonal Sur y fachada sobre Diagonal Sur con análisis compositivo

\section{Las regulaciones de fachada}

La diagonal Sur y la Diagonal Norte son avenidas que nacieron con un código postulativo. El referente más claro de ese código es el de los Boulevard de París planificados por Haussmann. EI SOMISA desarrolla una interpretación de estas exigencias, respetando las alturas de los niveles y los retiros, rediseñando la dialéctica entre el muro y los aventanamientos con la carpintería de metal y vidrio, acentuando los ritmos de separación entre el basamento el desarrollo y el remate con la estructura metálica y reinterpretando el concepto de esquina mediante la definición de un cuerpo compositivo resaltado con el empleo de los pórticos metálicos, el voladizo de esquina y el helipuerto que reemplaza la cúpula normalmente empleada en estos casos.

\section{Los elementos de composición y las jerarquías}

La fachada sobre Diagonal Sur presenta una organización de sus elementos que define tres sectores: el tramo próximo a la esquina con su exoestructura monumental, el tramo próximo al límite con el lote contiguo básicamente definido por la carpintería vidriada y un vacío entre ambos tramos que está definido por la misma carpintería y tres puentes de conexión entre los dos sectores mencionados anteriormente. Los tres sectores pueden ser entendidos como tres elementos compositivos de la fachada. De los tres, el tramo próximo a la esquina es el sector 


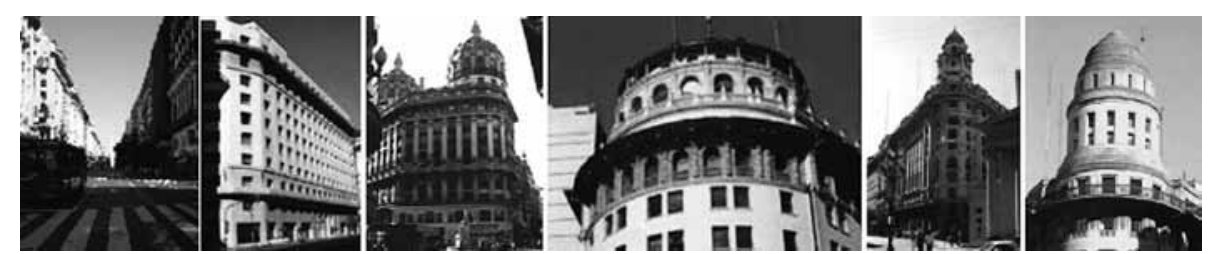

Figura $14 \mathrm{~A}$

Tejido de cuadra y esquinas de la Diagonal Norte.
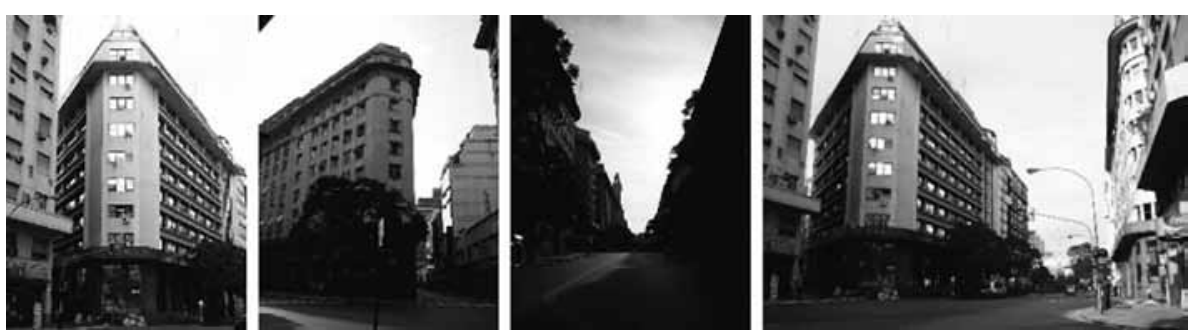

Figura 14B

Diagonal Sur: Dos esquinas, foto en dirección a Plaza de Mayo y foto en dirección a Avenida Belgrano.
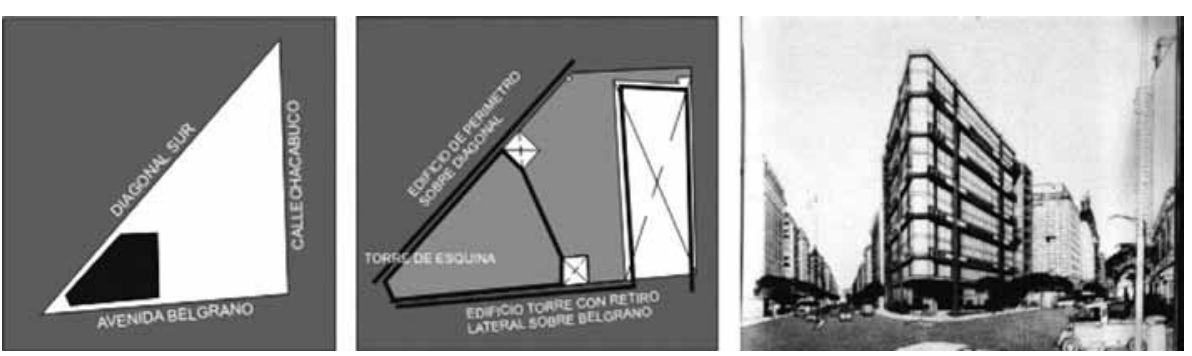

Figura 15

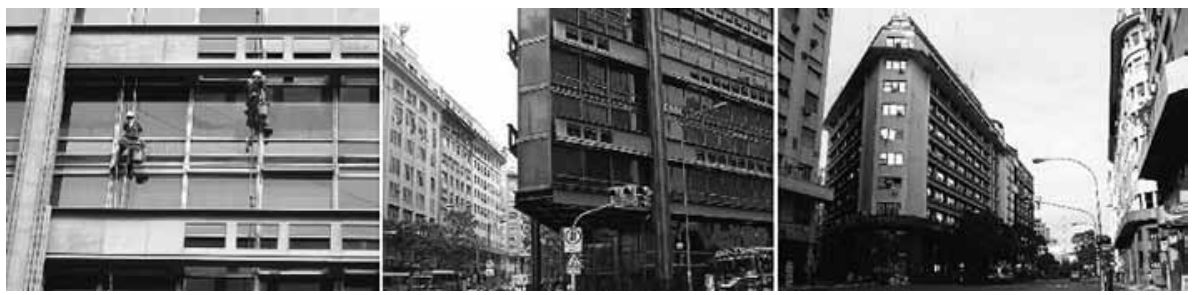

Figura 16

Analogía entre el orden de ritmos de las corniza, la relación entre sistema columnario y sistema murario clasicista y el orden tecnológico de estructura y cerramiento del SOMISA. más jerarquizado, ya que la exoestructura sólo aparece en su sector y es claramente el recurso más explícito de la naturaleza tecnológica de todo el edificio. Al quedar entonces un elemento muy jerarquizado definiendo la llegada a la esquina, un vacío o separación y un elemento más neutro como finalización del edificio al entregarse éste al resto de la cuadra, resulta posible imaginar que la retórica compositiva que se desarrolló continua con la tradición compositiva del bloque urbano en que las dos esquinas de la cuadra son jerarquizadas con un elemento de composición y el tramo que las une actúa como el desarrollo de algún edificio de composición clasicista en el que se alternan ejes y tramos en función de alguna simetría.

\section{Las similitudes morfológicas y rítmicas con el edificio vecino}

Existe una relación analógica clara entre la solución de los paños vidriados, el ancho y función de los pórticos y la organización de elementos de composición verticales de SOMISA y los del edificio de Vialidad Nacional lindero.

\section{La esquina y su remate}

Dentro de la estrategia compositiva mencionada en el punto anterior, la esquina es un sector clave. En el SOMISA, se recrea un trapecio simétrico que recupera la simetría que el lote no tiene, y hacer que se corresponda con la traza del lote un elemento de composición que simboliza la torre moderna que aunque fuera de los catálogos de elementos compositivos de remate de esquina provistos por la tradición académica, igualmente permite lograr el remate. Surge así una esquina que se presenta desmaterializada ya que sus elementos principales, el sistema exoestructural, se presentan separados en el punto de rotación de una cuadra respecto de la otra, dejando una ochava de vidrio que sugiere el vacío. Otro elemento importante en este remate de esquina es el helipuerto; el que hace el relevo de la cúpula habitualmente utilizada en estos casos.

\section{Los elementos de arquitectura y sus escalas monumentales}

Existe una analogía precisa entre los elementos que definen la estructura y el cerramiento en el edificio y la tradición de la superposición entre el sistema columnario y el sistema murario de la tradición clasicista. La exoestructura y la carpintería del cerramiento se vuelven análogas respectivamente al orden monumental columnario y al trazado del sistema murario. Es este paralelo jerárquico el 

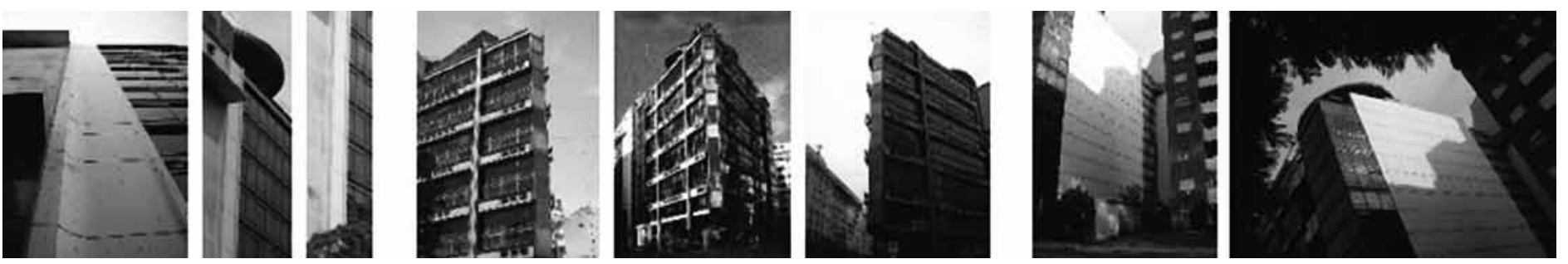

Figura 17

Articulaciones y límites: Lote lindero en Diagonal Sur, esquina y lote lindero en Avenida Belgrano
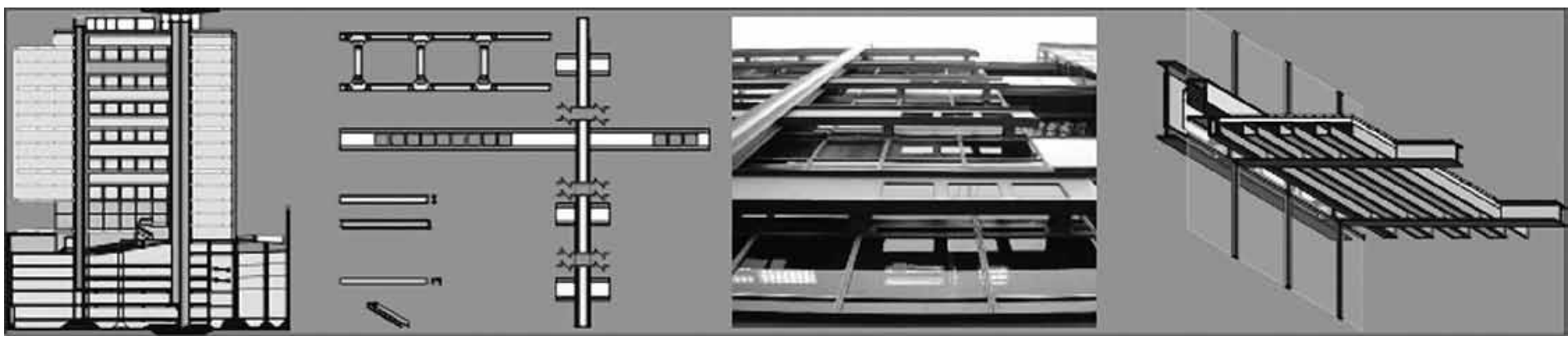

Figura 18

SOMISA; Sistema estructural y de carpinterías.

que hace que el tramo próximo a la esquina sea el «más importante», ya que posee los dos sistemas superpuestos.

\section{La conclusión de los límites del edificio}

Se logra por medio de una pieza vertical de hormigón armado con sección «C» que genera un hiato entre el SOMISA y el vecino en la Diagonal Sur y por medio del retiro que genera un vacío entre el SOMISA y el vecino en la Avenida Belgrano. La esquina actúa como sector de terminación en ambas cuadras y como elemento principal y central en la visión principal desde la avenida Belgrano.

\section{La estructura formal de Torre exenta}

\section{La ilusión de lo exento}

La descripción de la estructura y el cerramiento permite verificar que la tecnología empleada es adecuada para la construcción de una torre, aunque en realidad el edificio no llega a ser una torre. A pesar de no serlo, el tratamiento tecnológico y los vacíos practicados
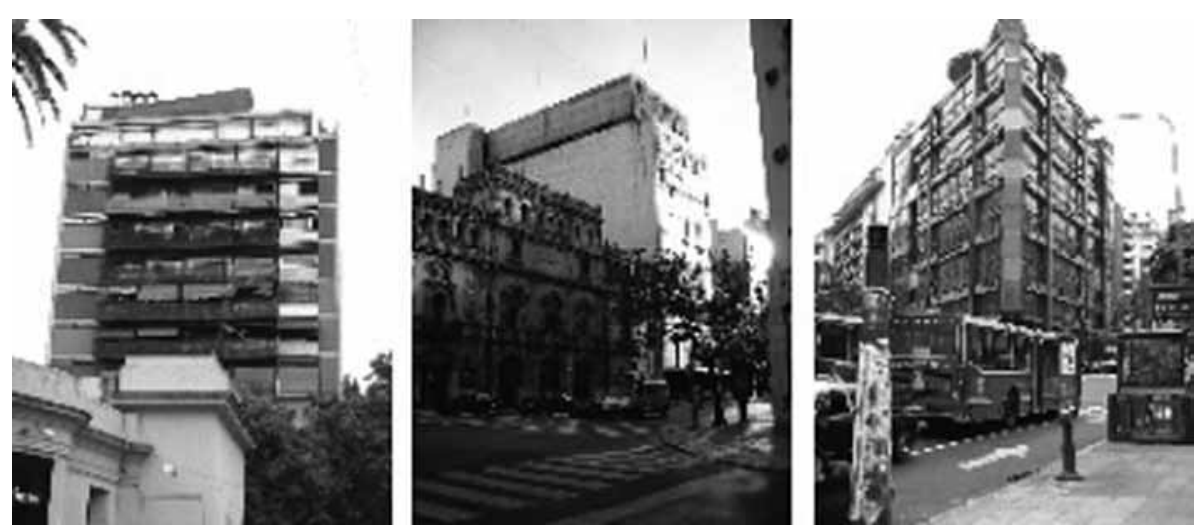

Figura 19

Edificio Posadas y Schiafino. Ampliación Teatro Nacional Cervantes y SOMISA. 
entre el elemento de la esquina y los otros elementos recrean la ilusión de la torre. En este sentido, la fachada sobre la Avenida Belgrano con el retiro respecto del lote lindero enfatiza aún más esta ilusión de torre exenta. Estos recursos ilusorios de estructura exenta han sido largamente practicados por Álvarez en varios edificios de vivienda y oficinas en altura durante la década de los 50 y los 60, y se puede afirmar que en el SOMISA Álvarez lleva el recurso al extremo; aún corriendo el riesgo de perder la síntesis.

\section{El concepto de figura y fondo}

Este recurso empleado por Álvarez anteriormente en varios de sus edificios, detectado por Marcelo Trabucco en su libro "Mario Roberto Álvarez» ${ }^{8}$ cuando analizaba el edificio de viviendas de Schiaffino y Posadas, fue empleado por Álvarez también en la ampliación del Teatro Nacional Cervantes, y vuelve a aparecer en el SOMISA. La manera en que se emplea es diferente de los casos anteriores en que existía una envolvente de naturaleza cóncava, en el SOMISA el fondo se aleja de la esquina, su arquitectura se va neutralizando en etapas, para llegar a los límites vecinos o como un vacío (Avenida Belgrano) o como una piel planar y sin accidentalidades (Diagonal Sur). Este recurso permite afianzar aún más la ilusión de la torre exenta.

\section{Atributos de la Torre moderna}

Posee tres accesos públicos, uno desde la esquina y dos opuestos entre sí y ubicados en correspondencia con los vacíos que separan los elementos principales en cada fachada.
Esta generosidad en su porosidad circulatoria es además de un recurso funcional, un recurso expresivo que magnifica la escala de los accesos y enfatiza una realidad de penetrabilidad propia de los objetos exentos. Los niveles desarrollados en sándwich actúan como fajas de transparencias y explicitación de actividades, sean éstas puntuales como en los sectores de planta baja y el último piso o sean éstas repetitivas como en todas las plantas tipo. El edificio se vuelve conceptualmente atravesable, haciendo que la fluidez espacial recree la realidad de la torre de cristal de origen miesiano.

\section{Expresión de una regularidad que reposa en el módulo}

Las distintas partes del edificio comparten una regularidad que se soporta en constantes funcionales y tecnológicas. El rigor tecnológico del acero, que impone una realidad del ensamble y terminación industrializada exige el empleo de un módulo que en este caso deriva de las medidas de las piezas de acero que SOMISA podía proveer. Otro aspecto derivado de la eficiencia en el funcionamiento administrativo hizo que fuera necesario compatibilizar el módulo tecnológico con el módulo determinado por la célula de trabajo en la oficina. Producto de ambas cuestiones, se adoptó un módulo general para el edificio de 3,76 metros, que se corresponde con la medida estándar de 1,22 × 3,70 del canalón de acero producido por SOMISA y el módulo de trabajo que resulta generoso respecto del módulo estándar de 1,50 metros por 1,50 metros que daría 3,00 por 3,00.

\section{Atenuación expresiva \\ de la realidad híbrida \\ de la composición}

Por medio de la homogeneidad de las texturas empleadas en la piel del edificio y por la presencia constante del trazado en todos los límites del edificio, se logró establecer una continuidad entre las partes similar a la continuidad expresiva que da el empleo de un material único, que en este caso produce una permanente descomposición y recomposición de la opacidad del límite causada por el impacto de la luz en las superficies vidriadas y metálicas.

\section{Bibliografía}

Bullrich, F. «El poder de la síntesis». Revista Summa 1987; 233/234.

Diez, F. Buenos Aires y algunas constantes en las transformaciones urbanas. Buenos Aires: Editorial UB, 1996.

Piñón, H. Mario Roberto Álvarez y Asociados. Barcelona: Edición UPC, 2002

Trabucco, M.A. Mario Roberto Álvarez. Buenos Aires: Instituto de Arte Americano de la Universidad de Buenos Aires, 1965.

Trabucco, M.A. ¿De la Totalidad a las Partes? Buenos Aires: Editorial de Belgrano, 1999.

Waisman, M. «El arte de ser simple en un mundo complicado». Revista Summa 1974; 80/81.

Trabucco, M.A. Mario Roberto Álvarez. Buenos

Aires: Instituto de Arte Americano de la Universidad de Buenos Aires, 1965 